\title{
A Hybrid Acoustic/Electromagnetic Technique for Locating Land Mines
}

\author{
Waymond R. Scott, Jr. ${ }^{a}$, Chistoph Schroeder ${ }^{\mathrm{a}}$, and James S. Martin ${ }^{\mathrm{b}}$ \\ ${ }^{\mathrm{a}} \mathrm{Sch}$ ool of Electrical and Computer Engineering \\ ${ }^{\mathrm{b}}$ School of Mechanical Engineering \\ Georgia Institute of Technology \\ Atlanta, GA 30332 \\ Phone: 404-894-3048 \\ EMail: waymond.scott@ece.gatech.edu
}

\begin{abstract}
A hybrid technique is presented that simultaneously uses both electromagnetic and acoustic waves in a synergistic manner to detect buried land mines. The system consists of an electromagnetic radar and an acoustic source. The acoustic source causes both the mine and the surface of the earth to be displaced. The electromagnetic radar is used to detect these displacements and, thus, the mine. An experimental and numerical model for the system has been developed.
\end{abstract}

\section{INTRODUCTION}

A technique to detect land mines that simultaneously uses both electromagnetic and acoustic waves in a synergistic manner is currently being investigated. The synergism has the potential to significantly enhance the signature of the mine with respect to the clutter and make it possible to detect a mine that would be impossible to detect by purely electromagnetic or purely acoustic means.

The configuration of the system currently being studied is shown in fig. 1. The system consists of an electromagnetic radar and an acoustic source. The acoustic source induces an acoustic (seismic) wave into the earth. The acoustic wave causes both the mine and the surface of the earth to be displaced. The displacement of the mine is different than the earth, because the acoustic properties of the mine are quite different than those of the earth. The displacement of the surface of the earth when a mine is present is different than when it is not present because of the waves scattered from the mine. The electromagnetic radar is used to detect these displacements and, thus, the mine. This idea has been discussed previously $[1,2]$, but it has not been seriously investigated.

In addition, the radar can be simultaneously used in a mode in which only the electromagnetic waves are used to identify (image) the mine. In this electromagnetic only mode, the radar is a conventional ground penetrating radar (GPR). Thus, both the acoustic and electromagnetic properties of the mine can be used to differentiate it from the earth. It may be possible to locate or identify mines with the

This work is supported in part by the US Army Research Office under contract DAAH04-96-1-0048.

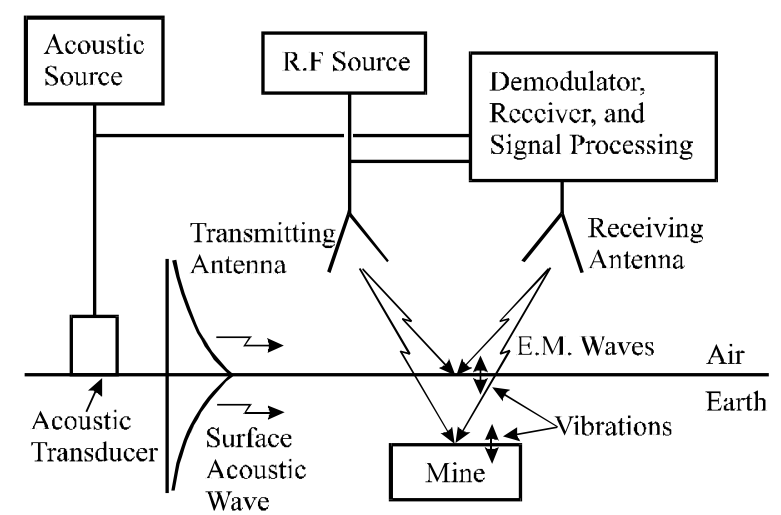

Fig. 1. Acousto-electromagnetic mine detection system with the acoustic transducer placed on the surface of the earth.

GPR that are invisible to the acoustic system or visa versa. Or it may me possible to combine the output of both the GPR and the acoustic system to lower the false alarm rate of the system.

An experimental model for the system has been constructed and automated. The experimental model is being used to demonstrate the viability of the technique and to study the interactions of the acoustic and electromagnetic waves with buried mines. The technique looks promising; we have been able to detect both simulated antipersonnel mines and antitank mines buried in damp sand. However, additional investigation of the technique is needed to determine the capabilities of the technique in more varied conditions. A two-dimensional finite-difference time-domain (FDTD) model for the acoustic waves has been also developed and is being used to help understand the interactions of the acoustic waves and the mines.

\section{EXPERIMENTAL MODEL}

A radar has been designed and built to measure the acoustic vibrations of the soil and the mine. The radar radiates electromagnetic waves that are reflected off of the vibrating interface. The reflected waves are received by the radar, and a Homodyne system is used to demodulate the signals. The vibrations are determined from these demodulated signals. The two biggest challenges to make this radar perform adequately for the mine detection system 
are 1) to make it sufficiently sensitive to be able to detect the small vibrations, and 2) to make the spot size (the area on the surface illuminated by the electromagnetic waves) sufficiently small. The radar can measure vibrations as small as $1 \mathrm{~nm}\left(10^{-9} \mathrm{~m}\right)$ as currently configured. To obtain this sensitivity, the radar was designed to minimize the effects of noise, such as the phase noise of the source and the electromagnetic interference from low-frequency magnetic fields. The spot size of the radar must be smaller than approximately one half of a wavelength of the acoustic waves. Currently, a small spot size is obtained by using an open-ended waveguide as the antenna for the radar. This antenna produces a sufficiently small spot size when the open end of the antenna is placed within a few centimeters of the surface. The results presented in this report are made with the radar operating at $8 \mathrm{GHz}$. The vibration of the surface of the sand has been measured with both the radar and an accelerometer; these measurements were compared and found to be in good agreement.

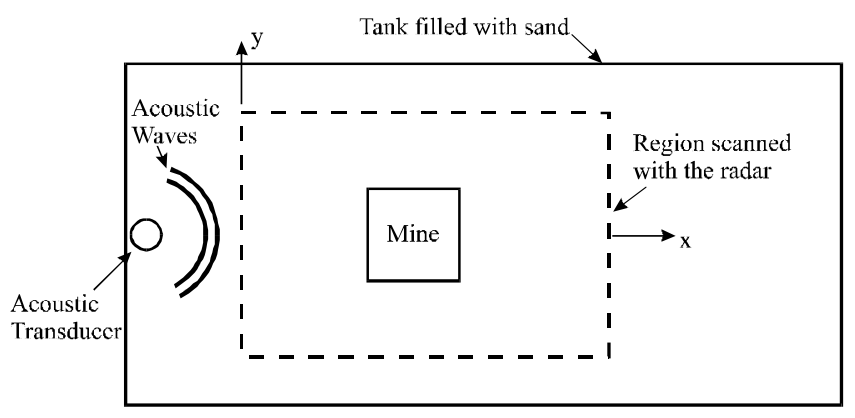

Fig. 2. Top view of experimental model.

A drawing of the top view of the experimental model is shown in fig. 2. The model consists of a tank that is filled with damp sand that has been packed to a relatively uniform density. The tank is approximately $120 \mathrm{~cm}$ wide, $120 \mathrm{~cm}$ deep, and $240 \mathrm{~cm}$ long. A transducer is placed on the surface of the sand and is used to launch the acoustic waves into the sand. The transducer is an electromagnetic shaker that is excited with a differentiated gaussian pulse. The pulse has a center frequency of $400 \mathrm{~Hz}$. The transducer has been adjusted so that it preferentially launches acoustic surface waves. These surface waves travel across the surface of the tank and interact with a mine that is buried in the sand. The electromagnetic radar is used to measure the vibrations caused by the acoustic waves. A x-y positioner is used to scan the radar over the surface of the sand. This system is under computer control, so it can scan the radar over the surface and record the data automatically. The region scanned with the radar is indicated on fig. 2. The vibrations are measured in this region as a function of time and position. The measurements are made on a uniform rectangular grid of discrete positions in the scanned region.

Pseudo color graphs of the amplitude of the displacement of the surface are presented in fig. 3 for three times. These results are for a simulated antitank mine buried in the sand.
The simulated anti-tank mine is made out of Acrylic plastic and is $30 \mathrm{~cm}$ wide, $30 \mathrm{~cm}$ long, and $7.5 \mathrm{~cm}$ high. The top of the mine is $5 \mathrm{~cm}$ below the surface of the sand. The position of the mine is indicated by the dotted white line. In these graphs, the color scale goes from black to blue to green to yellow to red to white. The smallest displacements are in black and the largest displacements are in white. At time \#1, the acoustic wave is seen traveling toward the mine. At time $\# 2$, the wave has reached the mine, and a portion of the wave

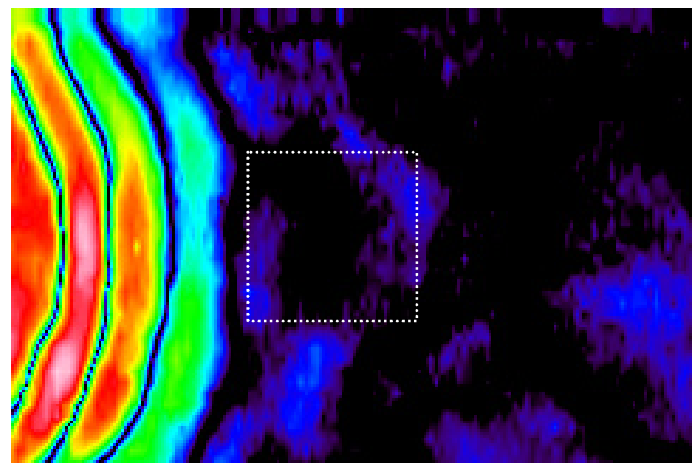

Time \#1 - Acoustic pulse traveling toward the mine

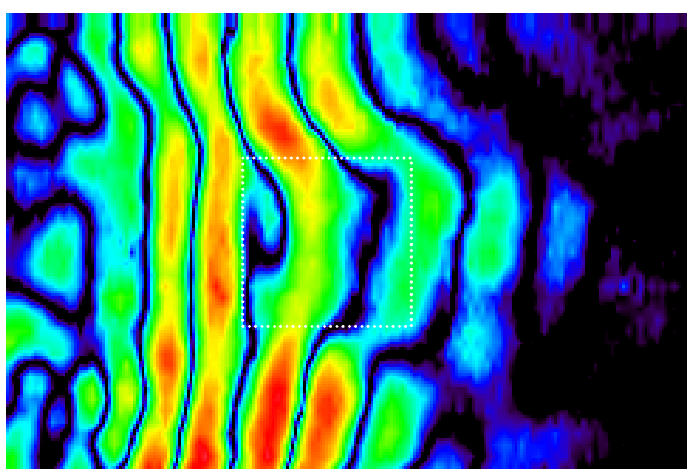

Time \#2 - Pulse interacting with the mine. The pulse is seen to travel faster across the mine.

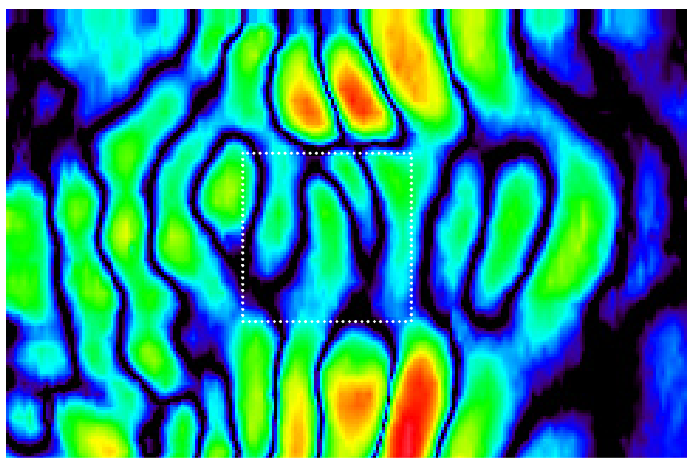

Time \#3 -Pulse is reflected from, transmitted through, and travels around the mine.

Fig. 3. Pseudo color graphs of the amplitude of the displacement of the surface of the sand for four times when a simulated antitank mine is buried in the sand. The top of the mine is $5 \mathrm{~cm}$ below the surface, and the mine is outlined by a white dotted line. 
is going across the mine while the rest is going around the mine. Notice that the portion of the wave that is going across the mine is ahead of the portion that is around the mine. This is because the mine is much stiffer than is the sand; thus, the wave appears to travel faster across the mine. The mine actually moves as if it is a rigid body. The motion of the mine is essentially a rocking motion excited by the wave motion in the sand. At time \#3, the wave is seen to be still going around the mine; however, a significant portion has passed across the mine. Notice that the wave that went across the mine is still ahead of the wave that went around the mine, and notice that the wave that went across the mine is smaller in amplitude than the one that went around the mine. Also notice that the displacements are smaller above the mine. The waves that are reflected from the mine can also be seen. The location and the shape of the mine are clearly evident in these graphs. We have also generated movies from the data recorded with the radar. In the movies, pseudo color graphs like those in fig. 3 are stored and played back sequentially. The interaction of the waves with the mine is clearer in the movies than it is in fig. 3; thus, is easier to see the mine.

\section{Numerical Model}

A two-dimensional FDTD model for the acoustic waves has been developed. Fig. 4 is a diagram of the FDTD model. The waves are injected with a point source, a perfectly matched layer (PML) is used to absorb the waves at the edge of the mesh, and a free surface boundary condition is used on the boundary between the soil and the air. The results from the numerical model are in fairly good agreement with those from the experimental model. The numerical model has been very useful in helping us to understand the interaction of the acoustic wave with the mines. With the numerical model, the waves can be observed below the surface; whereas, with the experimental model, the waves can only be observed on the surface.

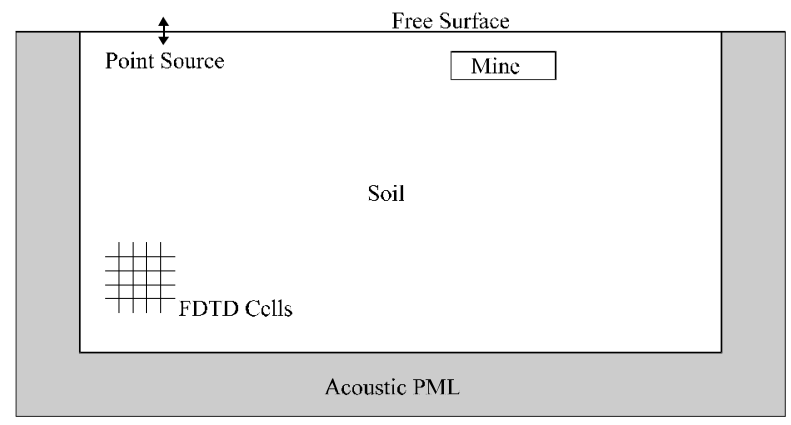

Fig. 4. Diagram of the acoustic FDTD model.

Pseudo color graphs of the amplitude of the velocity of the particles in the soil are presented in fig. 5 for the anti-tank mine buried $5 \mathrm{~cm}$ deep. The incident pulse is a differentiated gaussian pulse with a center frequency of $400 \mathrm{~Hz}$. The soil models the sand that is used in the experimental model. At time \#1, pressure $(\mathrm{P})$, shear $(\mathrm{S})$, and surface $(\mathrm{R})$ waves are seen to be launched. The shear and surface waves overlap, because they propagate at approximately the same velocity (the surface wave propagates slightly slower than the shear wave). The surface wave is the more intense wave near the surface. The pressure wave is ahead of the surface/shear wave because it propagates faster. At time \#2, the surface/shear wave is seen to have reached the mine. The portion of the wave that passed across the mine is seen to be ahead of the portion that is passing around the mine. Again this is because the wave travels faster in the mine than in the sand. Scattered pressure, shear, and surface waves are seen propagating away from the mine. Notice that a surface wave appears to be trapped above the mine, we have observed similar behavior in some of the experimental results.

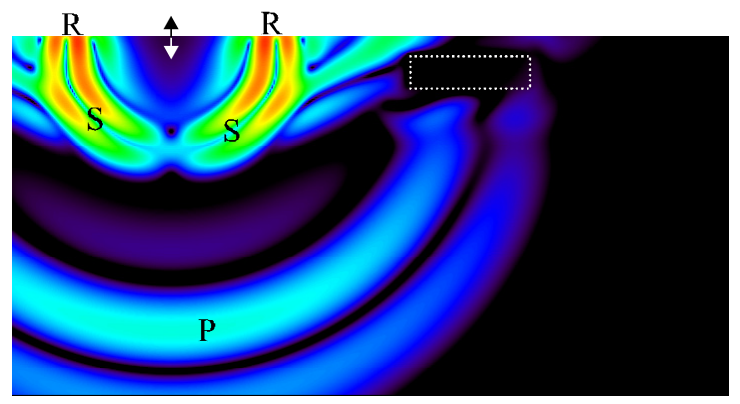

Time \#1 - Surface/shear wave has not reached the mine.

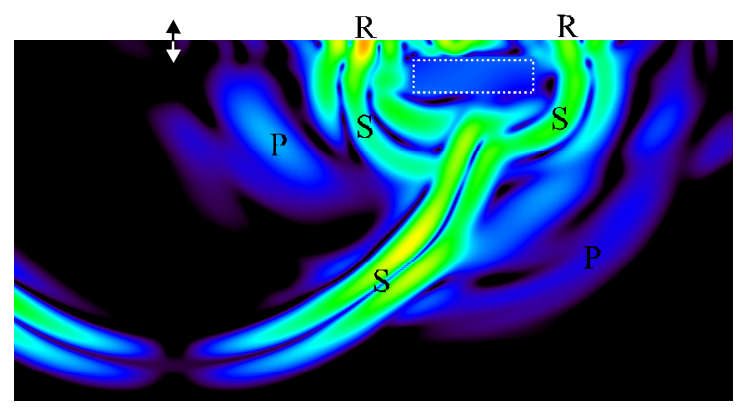

Time \#2 - Surface/shear wave is interacting with the mine.

Fig. 5. Pseudo color graphs of the amplitude of the velocity of the particles in the soil for four times when a simulated antitank mine is buried in the sand. The top of the mine is $5 \mathrm{~cm}$ below the surface, and the mine is outlined by a white dotted line.

\section{REFERENCES}

[1] G.S. Smith, "Summary Report: Workshop on New Directions for Electromagnetic Detection of Non-Metallic Mines," Report for U.S. Army BRDEC and ARO, June 1992

[2] C. Stewart, Summary of Mine Detection Research, Vol. I, pp. 172-179, Tech. Report 1636-TR, May 1960, U.S. Army Engineering Res. and Devel. Labs, Corps. of Eng., Belvoir, VA. 